Edited by:

Per J. Sjöström, The Research Institute of the McGill University Health Centre,

Canada

\section{Reviewed by:}

Jack Mellor, University of Bristol, United Kingdom

Alfredo Kirkwood, Johns Hopkins

University, USA

${ }^{*}$ Correspondence:

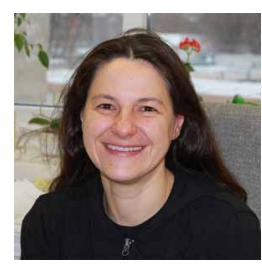

Elke Edelmann is a postdoctoral fellow at the Institute of Physiology,

Otto-von-Guericke University Magdeburg. She is interested in synaptic plasticity, neuromodulators (e.g., Dopamine), and BDNF at distinct hippocampal synapses.

elke.edelmann@med.ovgu.de

\title{
Dopamine regulates intrinsic excitability thereby gating successful induction of spike timing-dependent plasticity in CA1 of the hippocampus
}

\author{
Elke Edelmann ${ }^{1 *}$ and Volkmar Lessmann ${ }^{1,2 *}$ \\ 1 Institute of Physiology, Medical School, Otto-von-Guericke University Magdeburg, Magdeburg, Germany \\ ${ }^{2}$ Center of Behavioral Brain Sciences, Magdeburg, Germany
}

Long-term potentiation (LTP) and long-term depression (LTD) are generally assumed to be cellular correlates for learning and memory. Different types of LTP induction protocols differing in severity of stimulation can be distinguished in CA1 of the hippocampus. To better understand signaling mechanisms and involvement of neuromodulators such as dopamine (DA) in synaptic plasticity, less severe and more physiological low frequency induction protocols should be used. In the study which is reviewed here, critical determinants of spike timing-dependent plasticity (STDP) at hippocampal CA3-CA1 synapses were investigated. We found that DA via D1 receptor signaling, but not adrenergic signaling activated by the $\beta$-adrenergic agonist isoproterenol, is important for successful expression of STDP at CA3-CA1 synapses. The DA effect on STDP is paralleled by changes in spike firing properties, thereby changing intrinsic excitability of postsynaptic CA1 neurons, and gating STDP. Whereas $\beta$-adrenergic signaling also leads to a similar (but not identical) regulation of firing pattern, it does not enable STDP. In this focused review we will discuss the current literature on dopaminergic modulation of LTP in CA1, with a special focus on timing dependent (t-)LTP, and we will suggest possible reasons for the selective gating of STDP by DA [but not noradrenaline (NA)] in CA1.

Keywords: dopamine, spike timing-dependent plasticity, action potential, $\beta$-adrenergic signaling, hippocampal slice

\section{INTRODUCTION}

Long-term potentiation (LTP) and long-term depression (LTD) are considered as neuronal substrates for learning and memory (see e.g., Whitlock et al., 2006). In comparison to high frequency stimulation or pairing protocols for LTP induction, and compared to prolonged low frequency stimulation induced LTD, spike timing-dependent plasticity (STDP) is dependent on only moderate numbers of repeated precisely timed single pre- and postsynaptic action potentials (APs) (for review see e.g., Duguid and Sjostrom, 2006; Caporale and Dan, 2008; Markram et al., 2011, 2012; Feldman, 2012). The availability of transmitter-type neuromodulators [such as DA, noradrenaline (NA), acetylcholine (ACh)] has been shown recently to crucially regulate STDP in different brain areas (Seol et al., 2007; Pawlak and Kerr, 2008; Zhang et al., 2009; Huang et al., 2012). Whereas these neuromodulators are known since a while to affect conventional LTP and LTD 


\section{LTP induction}

Long-term potentiation (LTP) can be induced with various patterns of stimulation. Most used protocols are high frequency stimulations (HFS) or theta burst stimulations (TBS) to induce LTP or low frequency stimulation (LFS) to induce Long-term depression (LTD). More relevant to analyze signaling cascades during LTP is the paring protocol (combination of presynaptic tetanus and postsynaptic depolarization) or the low frequency stimulation protocol STDP.

Spike timing-dependent plasticity Paradigm to induce synaptic plasticity. STDP is dependent on precise timed activation of pre- and postsynaptic cells. Physiological relevant amount of activation can be used to induce long-lasting plastic changes in synaptic transmission. This protocol works in patch clamp configuration and allows access to single postsynaptic cell.

\section{Neuromodulators}

Substances acting as neurotransmitters and modulate actions of multiple neurons and synapses. Most of those substances activate G-protein coupled receptors and alter synaptic transmission or ionic conductions. Examples for neuromodulators: dopamine (DA), noradrenaline (NA), acetylcholine (ACh), and serotonin (5-HT).

Timing dependent ( $t-)$ LTP Timing-dependent ( $\mathrm{t}-$ ) LTP and LTD can be induced by STDP protocols. For t-LTP, presynaptic neurons have to be activated first, followed by the activation of postsynaptic neurons with ms scale delay. To evoke t-LTD, first postsynaptic neurons than presynaptic neurons are activated.

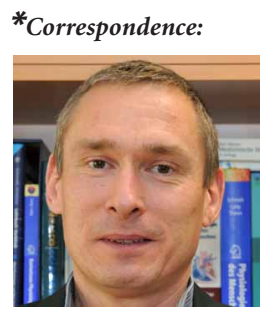

Volkmar Lessmann is professor for Physiology at the Medical Faculty of the Otto-von-Guericke University Magdeburg. His interests are focused on molecular and cellular mechanisms of synaptic plasticity in hippocampus, neocortex, and amygdala. A special focus of current experiments is on BDNF and Dopamine actions in STDP, and on mechanisms of BDNF secretion. lessmann@med.ovgu.de (for a recent review see e.g., Lisman et al., 2011), their cellular and molecular mechanisms of action in regulating timing dependent $(t-)$ LTP and $\mathbf{t}$-LTD, respectively, has just started to be investigated. In comparison to STDP in neocortex and striatum, the determinants of STDP in CA1 of hippocampal slices are less clear (see e.g., Buchanan and Mellor, 2010; Edelmann and Lessmann, 2011). In this focused review we will discuss the role of neuromodulators (especially DA) in the hippocampus for establishing STDP in the CAl subfield of the hippocampus.

\section{DESCRIPTION OF ORIGINAL DISCOVERY}

To investigate STDP in the CA1 region, we applied whole cell patch clamp recordings from pyramidal neurons of acute hippocampal slices of rats and mice (for details, see Edelmann and Lessmann, 2011). Briefly, STDP was induced by extracellular stimulation of the Schaffer collaterals with a STDP paradigm consisting of one presynaptic and (mostly) one postsynaptic AP stimulation with a time delay of +5 to $+10 \mathrm{~ms}$. This stimulation was repeated 100 times every $2 \mathrm{~s}$ to induce timing-dependent ( $\mathrm{t}-$ )LTP. We employed two different standard methods for slice preparation, using either sucrose-free artificial cerebrospinal liquid (ACSF), or sucrose containing ACSF (SUC). Using ELISA quantification our previous results revealed depletion of endogenous DA, selectively in SUC treated slices. Where indicated, DA levels were restored by bath application of exogenous DA $(20 \mu \mathrm{M})$, or action of endogenous DA was blocked by application of a specific D1 receptor antagonist (10 $\mu \mathrm{M}$ SCH23390). Parallel to changes in synaptic plasticity we also monitored resting membrane potential and AP firing properties of the neurons. To analyze the specificity of DA effects on STDP, we applied in some experiments the $\beta$-adrenergic agonist isoproterenol (ISO, $10 \mu \mathrm{M}$ ).

Using a STDP protocol with pairings of single pre- and postsynaptic spikes in CA1 pyramidal neurons of the hippocampus, our results revealed efficient induction and high amplitudes (on average $70 \%$ potentiation) of t-LTP when slices were prepared in ACSF solution, whereas preparation of slices in SUC containing solution strongly reduced the efficiency to induce STDP thereafter. ELISA measurements revealed reduced DA levels (to $\sim 60 \%$ of ACSF slices) in SUC prepared slices. In parallel with reduced synaptic plasticity, spike rise times, firing latencies and accommodation properties during repetitive spiking, as well as rise times of APs, elicited by brief high amplitude current injections during STDP induction, were significantly changed in CA1 pyramidal neurons upon depletion of endogenous DA. Application of exogenous DA $(20 \mu \mathrm{M})$ for $10-20$ min restored all AP properties and also completely rescued t-LTP under these conditions, back to values as observed in ACSF control slices (Figure 1). Similar to DA, also application of ISO $(10 \mu \mathrm{M})$, which has been shown previously to modulate backpropagating APs (Hoffman and Johnston, 1999), changed spike rise times (but not latency or accommodation). However, ISO was unable to rescue STDP (Figure 1). Interestingly, inhibition of D1 receptors in ACSF control slices inhibited t-LTP and also prolonged AP rise times (Figure 2). Overall, these results suggest that ambient levels of endogenous DA in hippocampal slices critically determine the efficiency of STDP induction in CA1, potentially by modulating properties of backpropagating APs during STDP induction (compare Edelmann and Lessmann, 2011).

\section{DISCUSSION \\ STDP IN THE HIPPOCAMPUS: UNDER WHICH CIRCUMSTANCES CAN t-LTP BE INDUCED IN CA1 PYRAMIDAL NEURONS?}

Cellular mechanisms of LTP are best investigated for high frequency activation or theta burst stimulation of pyramidal neurons in CA1 of the hippocampus (Bliss and Collingridge, 1993; Malenka and Nicoll, 1999). The neuromodulator DA has been described to modulate the efficacy of high frequency stimulation induced LTP in CA1 of the hippocampus via D1/D5 receptor signaling (see e.g., Yang et al., 2002; Lisman and Grace, 2005; Swant and Wagner, 2006).

Because of the clear learning rules for the induction, and the limited numbers of stimulated presynaptic (usually $<5$ ) and postsynaptic (just 1) neurons, STDP is ideally suited to investigate the molecular mechanisms of synaptic plasticity in CA1 at the single cell level. STDP is dependent on precise timing of single presynaptic and postsynaptic APs at low frequency and repetition number, rather than on excessive repetitive synaptic activation (reviewed in e.g., Bi and Poo, 2001; Duguid and Sjostrom, 2006; Markram et al., 2011, 2012; Feldman, 2012). Positive timing (i.e., presynaptic activation precedes postsynaptic firing of an AP) leads to a t-LTP, while t-LTD is typically achieved if postsynaptic spikes repeatedly precede excitatory postsynaptic potentials (see e.g., Markram et al., 1997; Bi and Poo, 1998). Most STDP studies have been performed in the cortex, because 

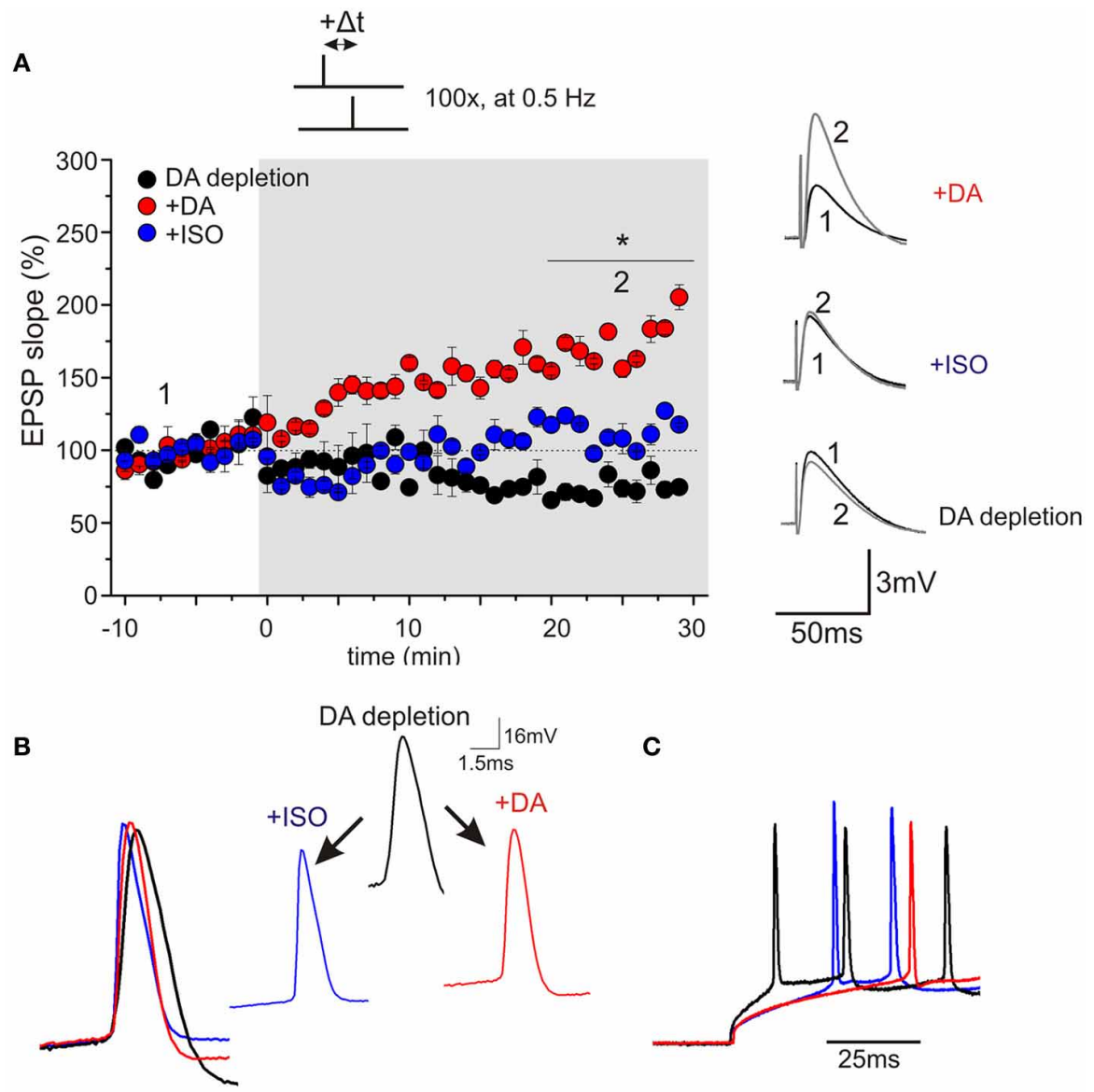

FIGURE 1 | Bath application of DA, but not ISO restores hippocampal spike-timing dependent plasticity in sucrose prepared slices. (A) Synaptic responses were recorded in the current clamp mode in CA1 pyramidal neurons of juvenile rats (P15-P20). Pre-stimulus membrane potential prior to recording was $-70 \mathrm{mV}$ for all cells. Presynaptic stimulation of Schaffer collaterals was performed every $20 \mathrm{~s}$ (for graphs: data binned to 1 min means). After recording of baseline for $10 \mathrm{~min}$, STDP was induced by a $1 \mathrm{EPSP} / 1 \mathrm{AP}$ pairing (100x, every $2 \mathrm{~s}$; at time point zero). Black symbols indicate neurons of the DA depleted group (DA depletion), red symbols indicate DA treated neurons (+20 $\mu \mathrm{M} \mathrm{DA})$ and blue symbols show ISO treatment $\left(+10 \mu \mathrm{M}\right.$ ISO; $\left.{ }^{*} p<0.05\right)$. Inset on the left side shows original traces before and after STDP induction for the three different conditions. (B) AP rise times for all three conditions. Rise time is significantly faster after application of the neuromodulators. (C) Modulation of spike latency by neuromodulators: latency to first spike for DA depletion (black), rescue by DA (red) and unsuccessful rescue by ISO (blue).

cortical synaptic connectivity allows for local paired recordings of connected pyramidal neurons more effectively than the hippocampus with CA3 and CA1 pyramidal neurons being connected over long distances. Furthermore, the required pre- and postsynaptic firing patterns for STDP induction in CA1 are not well understood (compare e.g., Magee and Johnston, 1997; Harvey and Svoboda, 2007; Tanaka et al., 2008; Sugisaki et al., 2011). In dissociated cultures of hippocampal neurons, and in CA1 pyramidal cells of cultured organotypic slices (which-however-lack intact neuromodulatory inputs) STDP is well established (e.g., Debanne et al., 1994, 1996; Bi and Poo, 1998;
Gerkin et al., 2007). In acute slices, STDP in CA1 is undisputed only in very young animals (Meredith et al., 2003, P9-P14 mice). For older, more mature preparations, the described learning rules in CA1 tend to be contradictory between different studies (for a recent review see Buchanan and Mellor, 2010). While Nishiyama et al. (2000) and Campanac and Debanne (2008) reported t-LTP after repetitive pairing of an EPSP with a single AP in CA1 of acute hippocampal slices from P26P33 or P15-P20 rats, respectively, neither Pike et al. (1999, young adult rats), Wittenberg and Wang (2006, P14-P28 rats), Remy and Spruston (2007, 3-5 weeks old rats), nor Carlisle et al. 
A

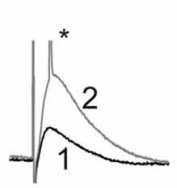

ACSF

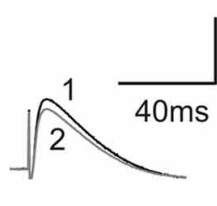

DA depletion

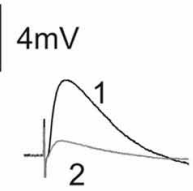

$\mathrm{ACSF}+\mathrm{SCH}$

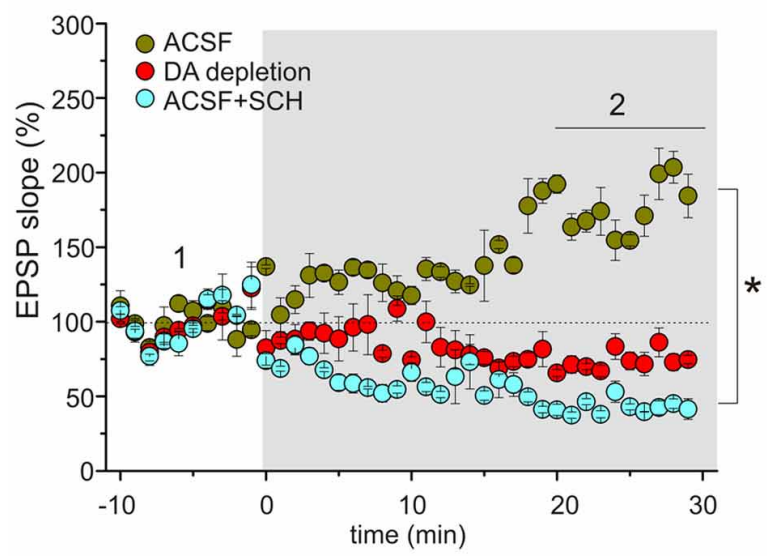

B
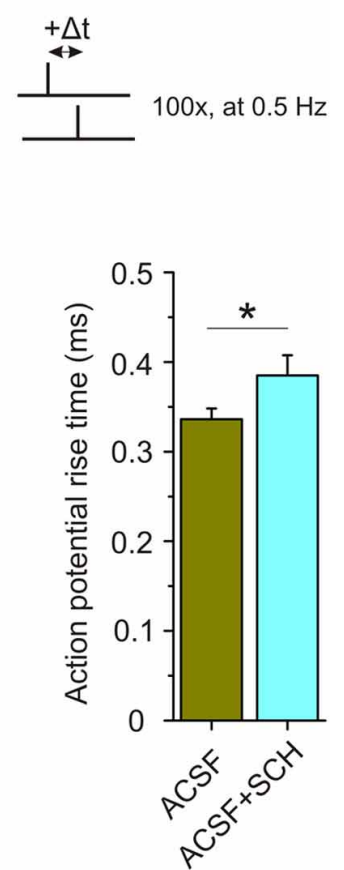

FIGURE 2 | Endogenous DA regulates STDP in CA1 of hippocampal slices via D1R signaling. (A) Bath application of the specific D1R antagonist SCH23390 $(10 \mu \mathrm{M})$ or DA depletion by preparation in SUC media inhibit t-LTP. For experimental conditions see Figure 1. Green symbols indicate average results for neurons of the ACSF group (ACSF), red symbols indicate results for DA depleted slices (DA depletion), and light blue symbols show results for neurons in ACSF prepared slices, treated with $\mathrm{SCH} 23390$ (+10 $\mu \mathrm{M} \mathrm{SCH}$ ). Inset above shows original traces before and after STDP induction for the three different conditions (small asterisk: action potential truncated). (B) AP rise time is significantly longer upon application of $\mathrm{SCH} 23390$ compared to ACSF $\left({ }^{*} p<0.05\right)$.

(2008, 4-12 months old mice) could show t-LTP with single spike pairing conditions in CA1. It can be argued that species and age differences could account for these divergent results. However, even with a given species and age, and employing a similar STDP protocol, different learning rules have been described (compare Wittenberg and Wang, 2006; Campanac and Debanne, 2008). In the light of recent studies indicating a regulation of STDP either by DA in the striatum (Pawlak and Kerr, 2008; Pawlak et al., 2010), by ISO and ACh in the neocortex (Seol et al., 2007), or by DA in dissociated hippocampal cultures (Zhang et al., 2009), and given our own results of DA-dependent modulation of STDP in CA1 of hippocampal slices (Edelmann and Lessmann, 2011), we hypothesize that the availability of specific neuromodulators critically determine the efficacy of STDP induction in the hippocampus. Thus, the previous discordant results (see above and Table $\mathbf{1}$ ) for t-LTP in CA1 might be explained on the basis of differential availability of endogenous neuromodulators in hippocampal slices used in these studies.

\section{NEUROMODULATION OF STDP G-protein dependent modulation of $t$-LTP and $t$-LTD}

Signaling via neuromodulators such as catecholamines (i.e., adrenaline, NA, DA) or $\mathrm{ACh}$ is known to be relevant for the efficacy of learning in vivo (e.g., Williams and Castner, 2006; Rossato et al., 2009), and for synaptic plasticity in vitro (e.g., Lisman and Grace, 2005; Scheiderer et al., 2008; Sara, 2009). Neuromodulator-dependent G-protein signaling regulates STDP in different brain areas.

In the visual cortex cooperation of different G-protein coupled receptors is necessary for functional bidirectional plasticity: exogenous ACh supports t-LTD via M1 receptors, whereas exogenously applied NA gates t-LTP via $\beta$-adrenergic receptors (Seol et al., 2007). The t-LTP promoting effect of NA relies on activation of $\beta$-receptors and subsequent Gs- and AC-signaling (Huang et al., 2012: but with pairing protocol instead of classical STDP; Salgado et al., 2012). Interestingly, while a high concentration of added NA enabled bidirectional 


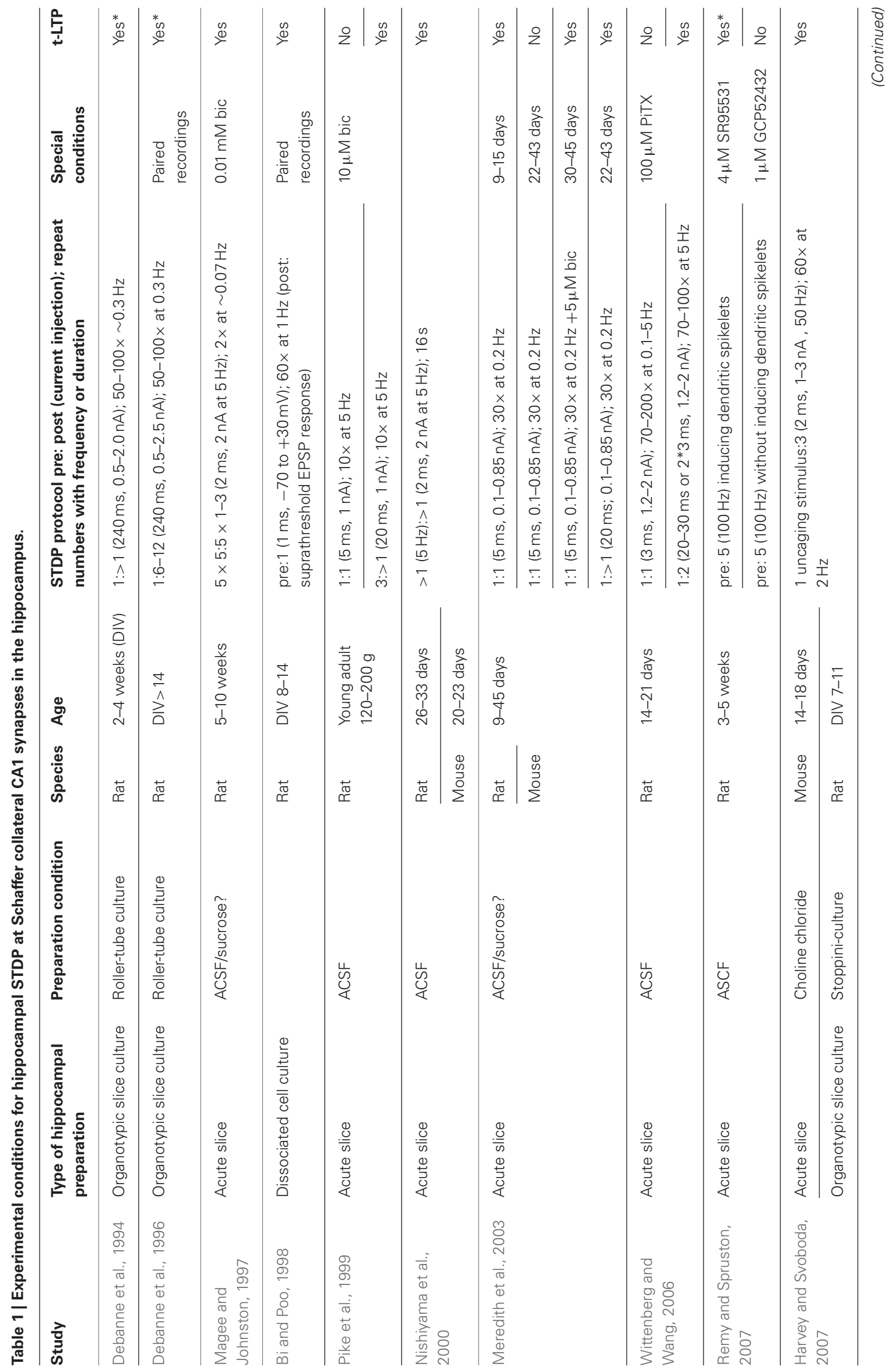




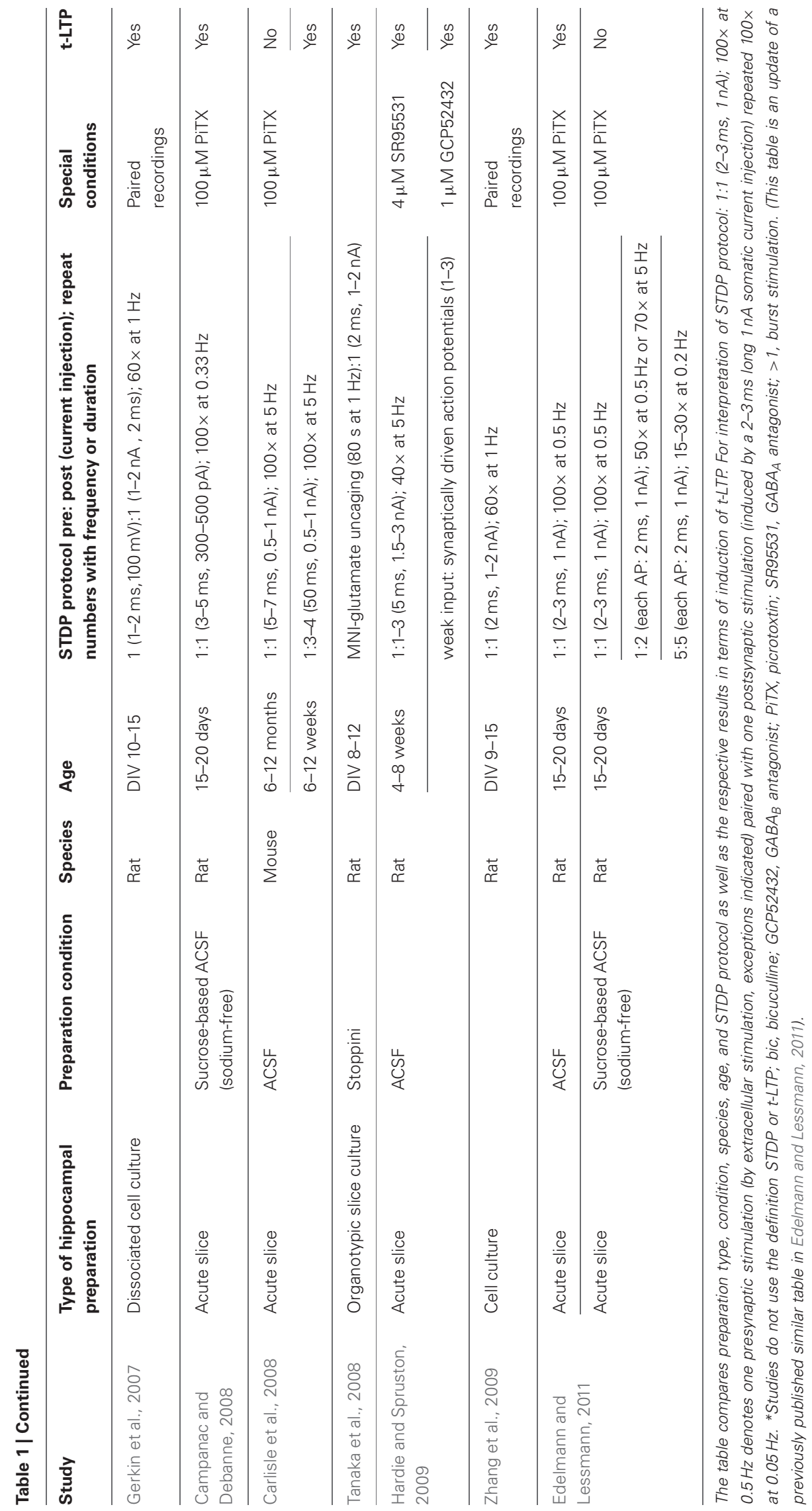


D1-like or D2-like receptor Dopamine (DA) acts on two main types of DA receptors. D1-like receptors (consistent of D1 and D5 receptors) are coupled to adenylate cyclase (AC) by G-protein $\alpha_{s}$. D1-receptor activation lead to activation of $\mathrm{AC}$ and subsequent cAMP and PKA activation. D2-like receptors (D2, D3, D4) are coupled to inhibitory G-protein $\left(\mathrm{G} \alpha_{\mathrm{i}}\right)$, which reduce cAMP level.

Backpropagation of action potentials Action potentials (APs) initiated at the axon hillock not only propagate into the axon, but also backpropagate into dendritic structures. Depending on the type of dendrite, this backpropagation is either regenerative (due to voltage gated channels along the dendrite) or decremental. In apical dendrites of CA1 pyramidal neurons, backpropagation is regulated by neuromodulators in a decremental fashion.
STDP, low concentration of NA lead to a depression-only state (Salgado et al., 2012). Muscarinergic and $\alpha 1$-adrenergic signaling via PLC- or Gq11 coupled receptors promote tLTD and suppress t-LTP in the visual cortex (Seol et al., 2007; Huang et al., 2012). In the prefrontal cortex activation of nAChRs on GABAergic interneurons by exogenous nicotine was shown to block t-LTP via $\mathrm{GABA}_{\mathrm{A}}$ receptor mediated attenuation of dendritic $\mathrm{Ca}^{2+}$ signals during t-LTP induction (Couey et al., 2007). Exogenous DA (via D2 receptors; D2R) enables t-LTP with intact GABAergic inhibition and prolongs effective spike timing windows for t-LTP by activation of D1/cAMP pathway (Xu and Yao, 2010). In the striatum, endogenous activation of D1/D5 receptors (D1/D5R) permits t-LTP and t-LTD, while inhibition of endogenous D2R signaling enables faster onset of potentiation in medium spiny projection neurons (MSNs) of the dorsolateral striatum (Pawlak and Kerr, 2008). In a related study, Shen et al. (2008) described distinct DA effects for a subpopulation of MSNs, which express D2R (but not D1R): for t-LTP, activation of the adenosine $\mathrm{A} 2$ receptor is necessary, while t-LTD can only be elicited after combined activation of the CB1 endocannabinoid receptor and D2 receptors. In the lateral amygdala, D2R activation by bath applied agonists permits t-LTP by decreasing feed forward inhibition (Bissiere et al., 2003), whereas in the ventral tegmental area (VTA) endogenous DA acts on D1 like receptors (most likely on D5R) to permit t-LTP in VTA DA cells (Argilli et al., 2008). Interestingly, the latter effect shares its signaling pathway with cocaine induced LTP in these neurons. Also in the hippocampus, neuromodulation of STDP is a common feature. Lin et al. (2003) reported an extended spike timing window for successful t-LTP induction after activation of $\beta$-adrenergic receptors, without affecting maximal t-LTP magnitude or tLTP induction at short positive timings. In dissociated hippocampal cell cultures, Zhang et al. (2009) showed that exogenous DA via $\mathrm{D} 1 \mathrm{R}$ activation prolonged the effective timing window for t-LTP, converted t-LTD to tLTP, and reduced the threshold for induction of t-LTP. In dentate gyrus granule cells, activation of $\mathrm{D} 1 \mathrm{R}$ by exogenous $\mathrm{DA}$ can reduce the threshold for t-LTP induction at medial perforant path-granule cell synapses in human and rat hippocampal slices (Hamilton et al., 2010; the authors used theta burst pairing instead of conventional spike timing protocols). At Schaffer collateral-CA1 synapses in acute rat hippocampal slices, D1R/D5R activation by endogenous DA is responsible for intact t-LTP at short positive pairings, and application of exogenous DA can rescue t-LTP after depletion of endogenous DA levels (Edelmann and Lessmann, 2011). However, activation of $\beta$-adrenergic receptors-although acting (like D1R/D5R) also via cAMP/PKA signaling-was not effective to restore t-LTP after DA depletion (Edelmann and Lessmann, 2011). For better comparison, the data discussed in this paragraph are summarized in Table 2 .

\section{Modulation of ionic conductances by DA}

Why is DA signaling essential for STDP? Neuromodulators such as DA have multiple effects on basal functions of neurons by different receptors coupled to different signaling cascades [D1-like or D2-like receptor (D1 or D2R), reviewed in Neve et al., 2004]. DA, acting on different subtypes of $\mathrm{DR}$, can either increase neuronal excitability by release of $\mathrm{Ca}^{2+}$ from intracellular $\mathrm{Ca}^{2+}$ stores and via cAMP dependent mechanisms (e.g., Benardo and Prince, 1982; Lezcano and Bergson, 2002), or it can decrease excitability by reducing peak sodium currents, by means of cAMP dependent phosphorylation of $\alpha$ subunits of voltage gated $\mathrm{Na}^{+}$channels (e.g., Cantrell et al., 1997). DA has also been described to either increase or decrease spike after hyperpolarization (e.g., Gribkoff and Ashe, 1984; Malenka and Nicoll, 1986; Berretta et al., 1990; Pedarzani and Storm, 1995), and to modulate further neuronal ion channels. Thus, DA increases the activity of $\mathrm{Ca}^{2+}$ activated $\mathrm{K}^{+}$channel (Benardo and Prince, 1982; Pockett, 1985), activates dendritic voltage dependent $\mathrm{K}^{+}$channels (Hoffman and Johnston, 1999; Johnston et al., 1999), increases h-channel activity (Rosenkranz and Johnston, 2006) and downregulates expression of t-type $\mathrm{Ca}^{2+}$ channels (Bender et al., 2010). Furthermore, DA can affect synaptic efficacy by changing frequency-dependent signal transmission via DA induced disinhibition (Ito and Schuman, 2008), or by enhancing net excitation by disinhibition of CA1 neurons via D3R (Hammad and Wagner, 2006). Since all of these DA-modulated voltage-dependent conductances contribute to shaping of APs, DA signaling likely modulates backpropagation of action potentials (bAP). This could account for the described DA-dependent alteration in the efficacy of STDP induction (compare section "G-protein dependent modulation of t-LTP and t-LTD”). 


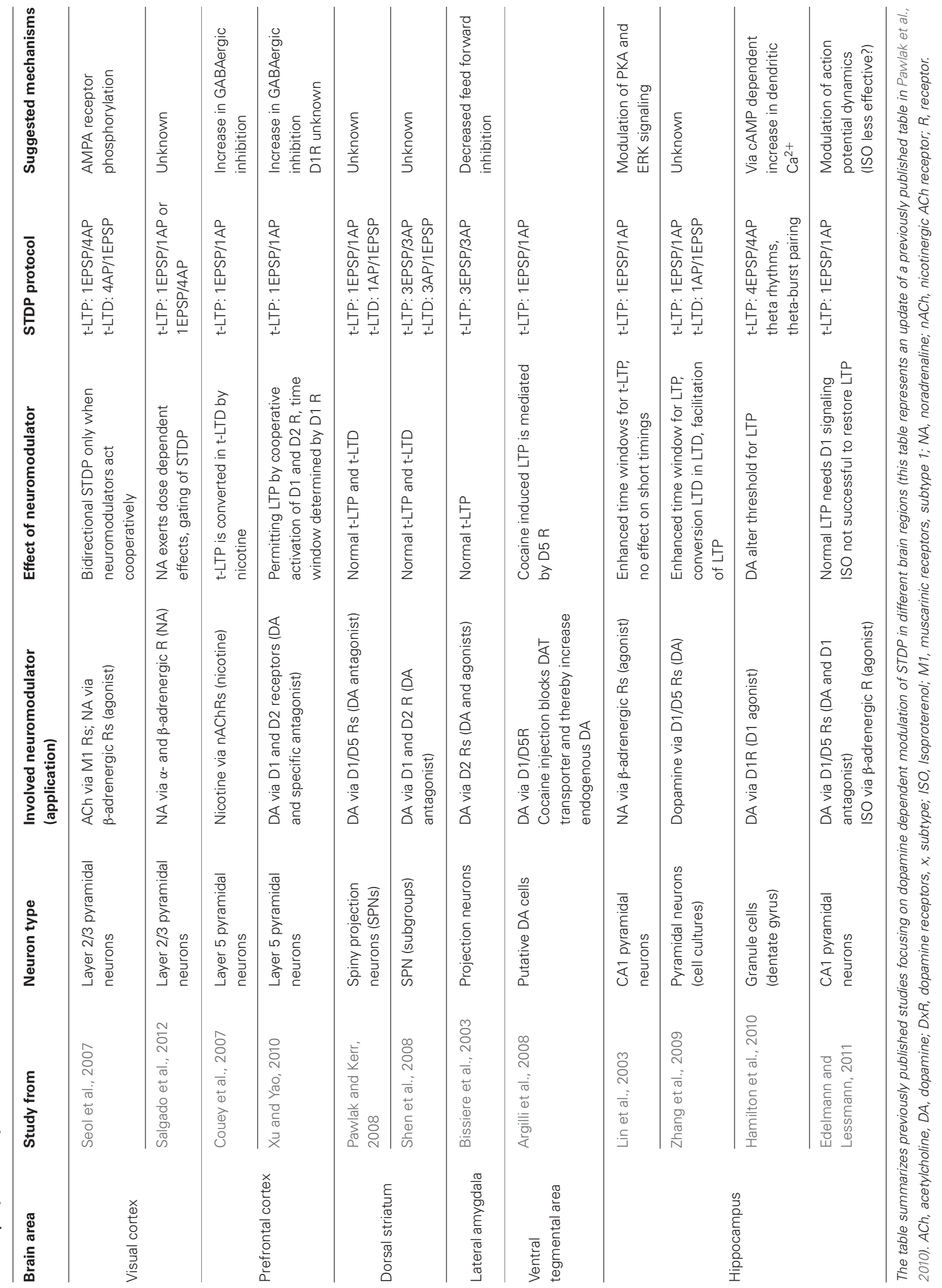




\section{Mechanism of DA regulated STDP in the hippocampus}

DA regulates conventional LTP in the hippocampus (reviewed in e.g., Lisman and Grace, 2005; Lisman et al., 2011). The main DAergic input to the hippocampus is delivered by VTA pathways (Gasbarri et al., 1994). DA via D1R activation can facilitate LTP induction by lowering the threshold to induce LTP (Li et al., 2003, 2011; Gao et al., 2006; Lemon and ManahanVaughan, 2006). Furthermore, endogenously released (by applying DA transporter antagonists) or exogenously applied DA, both, increase LTP in hippocampal slices (Swant and Wagner, 2006).

In our study (Edelmann and Lessmann, 2011), acute application of DA restored hippocampal STDP after DA depletion ( $60 \%$ of control) during preparation of slices (see Figure 1). In parallel with the absence of functional STDP, we also observed changed firing behavior of postsynaptic pyramidal cells: latency to first AP was reduced, spike rise times were prolonged, and AP frequency was enhanced in DA depleted slices. Following application of DA, also AP parameters were completely restored. The DA application dependent changes in AP properties that we observed were most likely a consequence of complex modulation of various ion channels [i.e.; voltage gated $\mathrm{Na}^{+}$currents, $\mathrm{I}_{\mathrm{h}}, \mathrm{K}^{+}$currents, Ttype voltage gated $\mathrm{Ca}^{2+}$ channels (compare section "Modulation of ionic conductances DA")]. Interestingly, application of ISO ( $\beta$-adrenergic agonist) also restored most of the AP properties in DA depleted slices, but did not restore impaired STDP in our experiments. The noradrenergic (NA) innervation of the hippocampus in vivo is derived from the locus coeruleus (e.g., Scheiderer et al., 2008; Sara, 2009). Similar to DA transmission originating from the VTA, NA inputs in acute hippocampal slices are truncated and only axon shafts including presynaptic terminals remain in this preparation, which are likely to release DA and NA either spontaneously or in response to extracellular stimulation used to activate CA1 synapses. Our observation that a D1R antagonist (SCH23390) changes spike properties and inhibits t-LTP in CA1 are consistent with DA being released in the slices and thereby affecting firing properties and t-LTP. Whether effects of DA on spiking and backpropagation of APs can account completely for inhibited t-LTP after DA depletion, remains to be determined.
While activation of $\beta$-adrenergic receptors with ISO can restore STDP via adenylate cyclase (AC) signaling in the visual cortex (Seol et al., 2007), a similar rescue of STDP could not be detected in hippocampal CA1-STDP for short positive pairings (Edelmann and Lessmann, 2011). However, Lin et al. (2003) described $\beta$-adrenergic prolongation of spike timing windows for t-LTP induction in CA1. Possibly we were unable to see a rescue effect due to the short positive pairings used in our experiments. Alternatively, differential coupling of receptors to downstream signaling cascades and/or differences in subcellular localization of D1 vs. NA receptors could account for these seemingly divergent results between DA and NA signaling in t-LTP in CA1 (see e.g., SwansonPark et al., 1999). It also seems possible that ISO concentrations which were sufficient for modulating firing of CA1 pyramidal neurons (Edelmann and Lessmann, 2011) were subthreshold for gating STDP. Thus, future analysis of dose response curves for DA and NA, respectively, should clarify whether DA and NA effects differ qualitatively or quantitatively in shaping t-LTP in CA1.

Differences in amplitude and time course of dendritic calcium waves might underlie the differential role of $\mathrm{DA}$ and NA, respectively, in gating STDP. Therefore, future calcium imaging in CA1 pyramidal neuron dendrites should be helpful to address this possible difference.

Importantly, only DA (but not ISO) changed AP latency and accommodation properties in our recordings from CA1 neurons, which could underlie the parallel gating effect of DA for STDP. Thus, future experiments investigating DA dependent modulation of $\mathrm{K}^{+}$conductances which regulate these firing properties might be able to explain the differential modulation of STDP by DA and NA.

In conclusion, STDP studies should be evaluated critically for experimental conditions (such as preparation methods, age and species of animals, type of preparation, stimulation paradigms) which could result in changed availability of neuromodulators such as DA, NA, and $\mathrm{ACh}$ in the slices. From our own results and from several recent studies in striatum, neocortex and hippocampus we conclude that seemingly subtle differences in experimental conditions (e.g., in the composition of preparation and/or recording media) can dramatically influence the success of STDP. With respect to the action of a specific neuromodulator in 
STDP it seems highly relevant to investigate effects on basal and active electrophysiological properties, since changing AP properties and backpropagation of APs by specific neuromodulator actions might underlie changes in STDP.

\section{ACKNOWLEDGMENTS}

This work was supported by grants from the DFG (SFB 779; LE 1020/2-1), and by the Schram Stiftung. The authors thank Dr. Thomas Endres and Efrain Cepeda for helpful discussions and critical reading of the manuscript.

\section{REFERENCES}

Argilli, E., Sibley, D. R., Malenka, R. C., England, P. M., and Bonci, A. (2008). Mechanism and time course of cocaine-induced longterm potentiation in the ventral tegmental area. J. Neurosci. 28, 9092-9100.

Benardo, L. S., and Prince, D. A. (1982). Dopamine modulates a Ca2+-activated potassium conductance in mammalian hippocampal pyramidal cells. Nature 297, 76-79.

Bender, K. J., Ford, C. P., and Trussell, L. O. (2010). Dopaminergic modulation of axon initial segment calcium channels regulates action potential initiation. Neuron 68, 500-511.

Berretta, N., Berton, F., Bianchi, R., Capogna, M., Francesconi, W., and Brunelli, M. (1990). Effects of dopamine, D-1 and D-2 dopaminergic agonists on the excitability of hippocampal CA1 pyramidal cells in guinea pig. Exp. Brain Res. 83, 124-130.

Bi, G. Q., and Poo, M. M. (1998). Synaptic modifications in cultured hippocampal neurons: dependence on spike timing, synaptic strength, and postsynaptic cell type. J. Neurosci. 18, 10464-10472.

Bi, G. Q., and Poo, M. M. (2001). Synaptic modification by correlated activity: Hebb's postulate revisited. Annu. Rev. Neurosci. 24, 139-166.

Bissiere, S., Humeau, Y., and Luthi, A. (2003). Dopamine gates LTP induction in lateral amygdala by suppressing feedforward inhibition. Nat. Neurosci. 6, 587-592.

Bliss, T. V., and Collingridge, G. L. (1993). A synaptic model of longterm potentiation in the hippocampus. Nature 361, 31-39.

Buchanan, K. A., and Mellor, J. R. (2010). The activity requirements for spike timing-dependent plasticity in the hippocampus. Front. Syn. Neurosci. 2:11. doi: 10.3389/fnsyn.2010.00011

Campanac, E., and Debanne, D. (2008). Spike timing-dependent plasticity: a learning rule for dendritic integration in rat CA1 pyramidal neurons. J. Physiol. 586, 779-793.

Cantrell, A. R., Smith, R. D., Goldin, A. L., Scheuer, T., and Catterall, W. A. (1997). Dopaminergic modulation of sodium current in hippocampal neurons via cAMP-dependent phosphorylation of specific sites in the sodium channel alpha subunit. J. Neurosci. 17, 7330-7338.

Caporale, N., and Dan, Y. (2008) Spike timing-dependent plasticity: a Hebbian learning rule. Annu. Rev. Neurosci. 31, 25-46.

Carlisle, H. J., Fink, A. E., Grant, S. G., and O'Dell, T. J. (2008). Opposing effects of PSD-93 and PSD-95 on long-term potentiation and spike timing-dependent plasticity. J. Physiol. 586, 5885-5900.

Couey, J. J., Meredith, R. M., Spijker, S., Poorthuis, R. B., Smit, A. B., Brussaard, A. B., et al. (2007). Distributed network actions by nicotine increase the threshold for spike-timing-dependent plasticity in prefrontal cortex. Neuron 54 73-87.

Debanne, D., Gahwiler, B. H., and Thompson, S. M. (1994) Asynchronous pre- and postsynaptic activity induces associative long-term depression in area CA1 of the rat hippocampus in vitro. Proc. Natl. Acad. Sci. U.S.A. 91, 1148-1152.

Debanne, D., Gahwiler, B. H., and Thompson, S. M. (1996). Cooperative interactions in the induction of long-term potentiation and depression of synaptic excitation between hippocampal CA3-CA1 cell pairs in vitro. Proc. Natl. Acad. Sci. U.S.A. 93, 11225-11230.

Duguid, I., and Sjostrom, P. J. (2006). Novel presynaptic mechanisms for coincidence detection in synaptic plasticity. Curr. Opin. Neurobiol. 16, 312-322.

Edelmann, E., and Lessmann, V. (2011). Dopamine modulates spike timing-dependent plasticity and action potential properties in CA1 pyramidal neurons of acute rat hippocampal slices. Front. Syn. Neurosci. 3:6. doi: 10.3389/fnsyn.2011.00006

Feldman, D. (2012). The spike timing dependence of plasticity. Neuron 75, 556-571.

Gao, C., Sun, X., and Wolf, M. E. (2006). Activation of D1 dopamine receptors increases surface expression of AMPA receptors and facilitates their synaptic incorporation in cultured hippocampal neurons. J. Neurochem. 98, 1664-1677.

Gasbarri, A., Verney, C., Innocenzi, R. Campana, E., and Pacitti, C. (1994). Mesolimbic dopaminergic neurons innervating the hippocampal formation in the rat: a combined retrograde tracing and immunohistochemical study. Brain Res. 668 , 71-79.

Gerkin, R. C., Lau, P. M., Nauen, D. W., Wang, Y. T., and Bi, G. Q. (2007). Modular competition driven by NMDA receptor subtypes in spike-timing-dependent plasticity. J. Neurophysiol. 97, 2851-2862.

Gribkoff, V. K., and Ashe, J. H. (1984). Modulation by dopamine of population responses and cell membrane properties of hippocampal CA1 neurons in vitro. Brain Res. 292, 327-338.

Hamilton, T. J., Wheatley, B. M. Sinclair, D. B., Bachmann, M. Larkum, M. E., and Colmers, W. F. (2010). Dopamine modulates synaptic plasticity in dendrites of rat and human dentate granule cells. Proc. Natl. Acad. Sci. U.S.A. 107, 18185-18190.

Hammad, H., and Wagner, J. J. (2006) Dopamine-mediated disinhibition in the CA1 region of rat hippocampus via D3 receptor activation. J. Pharmacol. Exp. Ther. 316, 113-120.

Hardie, J., and Spruston, N. (2009). Synaptic depolarization is more effective than back-propagating action potentials during induction of associative long-term potentiation in hippocampal pyramida neurons. J. Neurosci. 29, 3233-3241.

Harvey, C. D., and Svoboda, K. (2007) Locally dynamic synaptic learning rules in pyramidal neuron dendrites. Nature 450, 1195-1200.

Hoffman, D. A., and Johnston, D. (1999). Neuromodulation of dendritic action potentials. J. Neurophysiol. 81, 408-411.

Huang, S., Trevino, M., He, K., Ardiles, A., Pasquale, R., Guo, Y., et al. (2012). Pull-push neuromodulation of LTP and LTD enables bidirectional experience-induced synaptic scaling in visual cortex. Neuron 73 , 497-510.

Ito, H. T., and Schuman, E. M (2008). Frequency-dependent signal transmission and modulation by neuromodulators. Front. Neurosci. 2:138-144. doi: 10.3389/neuro.01.027.2008

Johnston, D., Hoffman, D. A., Colbert, C. M., and Magee, J. C. (1999). Regulation of back-propagating action potentials in hippocampal neurons. Curr. Opin. Neurobiol. 9, 288-292.

Lemon, N., and Manahan-Vaughan, D. (2006). Dopamine D1/D5 receptors gate the acquisition of novel information through hippocampal long-term potentiation and longterm depression. J. Neurosci. 26, 7723-7729.

Lezcano, N., and Bergson, C. (2002). D1/D5 dopamine receptors stimulate intracellular calcium release in primary cultures of neocortical and hippocampal neurons. J. Neurophysiol. 87, 2167-2175.

Li, C., Dabrowska, J., Hazra, R., and Rainnie, D. G. (2011). Synergistic activation of dopamine D1 and TrkB receptors mediate gain control of synaptic plasticity in the basolateral amygdala. PLoS ONE 6:e26065. doi: 10.1371/journal.pone.0026065

Li, S., Cullen, W. K., Anwyl, R., and Rowan, M. J. (2003). Dopaminedependent facilitation of LTP induction in hippocampal CA1 by exposure to spatial novelty. Nat. Neurosci. 6, 526-531.

Lin, Y. W., Min, M. Y., Chiu, T. H., and Yang, H. W. (2003). Enhancement of associative long-term potentiation by activation of beta-adrenergic receptors at CA1 synapses in rat hippocampal slices. J. Neurosci. 23, 4173-4181.

Lisman, J., Grace, A. A., and Duzel, E. (2011). A neoHebbian framework for episodic memory; role of dopamine-dependent late LTP. Trends Neurosci. 34, 536-547.

Lisman, J. E., and Grace, A. A. (2005). The hippocampal-VTA loop: controlling the entry of information into long-term memory. Neuron 46 703-713.

Magee, J. C., and Johnston, D. (1997). A synaptically controlled, associative signal for Hebbian plasticity in hippocampal neurons. Science 275 209-213.

Malenka, R. C., and Nicoll, R. A. (1986). Dopamine decreases the 
calcium-activated afterhyperpolarization in hippocampal CA1 pyramidal cells. Brain Res. 379, 210-215.

Malenka, R. C., and Nicoll, R. A. (1999). Long-term potentiation: a decade of progress? Science 285, 1870-1874.

Markram, H., Gerstner, W., and Sjostrom, P. J. (2011). A history of spike-timing-dependent plasticity. Front. Syn. Neurosci. 3:4. doi: 10.3389/fnsyn.2011.00004

Markram, H., Gerstner, W., and Sjostrom, P. J. (2012). Spiketiming-dependent plasticity: a comprehensive overview. Front. Syn. Neurosci. 4:2. doi: 10.3389/fnsyn.2012.00002

Markram, H., Lubke, J., Frotscher, M., and Sakmann, B. (1997). Regulation of synaptic efficacy by coincidence of postsynaptic APs and EPSPs. Science 275, 213-215.

Meredith, R. M., Floyer-Lea, A. M., and Paulsen, O. (2003). Maturation of long-term potentiation induction rules in rodent hippocampus: role of GABAergic inhibition. J. Neurosci. 23, 11142-11146.

Neve, K. A., Seamans, J. K., and Trantham-Davidson, H. (2004). Dopamine receptor signaling. J. Recept. Signal Transduct. Res. 24, 165-205.

Nishiyama, M., Hong, K., Mikoshiba, K., Poo, M. M., and Kato, K. (2000). Calcium stores regulate the polarity and input specificity of synaptic modification. Nature 408, 584-588.

Pawlak, V., and Kerr, J. N. (2008). Dopamine receptor activation is required for corticostriatal spike-timing-dependent plasticity. J. Neurosci. 28, 2435-2446.

Pawlak, V., Wickens, J. R., Kirkwood, A., and Kerr, J. N. D. (2010). Timing is not everything: neuromodulation opens the STDP gate. Front. Syn. Neurosci. 2:146. doi: 10.3389/fnsyn.2010.00146

Pedarzani, P., and Storm, J. F. (1995). Dopamine modulates the slow
$\mathrm{Ca}(2+)$-activated $\mathrm{K}+$ current IAHP via cyclic AMP-dependent protein kinase in hippocampal neurons. J. Neurophysiol. 74, 2749-2753.

Pike, F. G., Meredith, R. M., Olding, A. W., and Paulsen, O. (1999). Rapid report: postsynaptic bursting is essential for "Hebbian" induction of associative long-term potentiation at excitatory synapses in rat hippocampus. J. Physiol. 518( $\mathrm{Pt} 2)$, 571-576.

Pockett, S. (1985). Dopamine changes the shape of action potentials in hippocampal pyramidal cells. Brain Res. 342, 386-390

Remy, S., and Spruston, N. (2007). Dendritic spikes induce singleburst long-term potentiation. Proc. Natl. Acad. Sci. U.S.A. 104, 17192-17197.

Rosenkranz, J. A., and Johnston, D. (2006). Dopaminergic regulation of neuronal excitability through modulation of Ih in layer $\mathrm{V}$ entorhinal cortex. J. Neurosci. 26, 3229-3244.

Rossato, J. I., Bevilaqua, L. R., Izquierdo, I., Medina, J. H., and Cammarota, M. (2009). Dopamine controls persistence of long-term memory storage. Science 325, 1017-1020.

Salgado, H., Kohr, G., and Trevino, M. (2012). Noradrenergic 'tone' determines dichotomous control of cortical spike-timing-dependent plasticity. Sci. Rep. 2:417. doi: 10.1038/srep00417

Sara, S. J. (2009). The locus coeruleus and noradrenergic modulation of cognition. Nat. Rev. Neurosci. 10, 211-223.

Scheiderer, C. L., Smith, C. C., McCutchen, E., McCoy, P. A., Thacker, E. E., Kolasa, K., et al. (2008). Coactivation of $\mathrm{M}(1)$ muscarinic and alphal adrenergic receptors stimulates extracellular signal-regulated protein kinase and induces long-term depression at CA3-CA1 synapses in rat hippocampus. J. Neurosci. 28, 5350-5358.
Seol, G. H., Ziburkus, J., Huang, S., Song, L., Kim, I. T., Takamiya, K., et al. (2007). Neuromodulators control the polarity of spike-timingdependent synaptic plasticity. Neuron 55, 919-929.

Shen, W., Flajolet, M., Greengard, P., and Surmeier, D. J. (2008). Dichotomous dopaminergic control of striatal synaptic plasticity. Science 321, 848-851.

Sugisaki, E., Fukushima, Y., Tsukada, M., and Aihara, T. (2011). Cholinergic modulation on spike timing-dependent plasticity in hippocampal CA1 network. Neuroscience 192, 91-101.

Swanson-Park, J. L., Coussens, C. M. Mason-Parker, S. E., Raymond, C. R., Hargreaves, E. L., Dragunow, M., et al. (1999). A double dissociation within the hippocampus of dopamine D1/D5 receptor and beta-adrenergic receptor contributions to the persistence of longterm potentiation. Neuroscience 92, 485-497.

Swant, J., and Wagner, J. J. (2006). Dopamine transporter blockade increases LTP in the CA1 region of the rat hippocampus via activation of the D3 dopamine receptor. Learn. Mem. 13, 161-167.

Tanaka, J., Horiike, Y., Matsuzaki, M., Miyazaki, T., Ellis-Davies, G. C., and Kasai, H. (2008). Protein synthesis and neurotrophindependent structural plasticity of single dendritic spines. Science 319, 1683-1687.

Whitlock, J. R., Heynen, A. J., Shuler, M. G., and Bear, M. F. (2006). Learning induces long-term potentiation in the hippocampus. Science 313, 1093-1097.

Williams, G. V., and Castner, S. A. (2006). Under the curve: critical issues for elucidating D1 receptor function in working memory. Neuroscience 139, 263-276.

Wittenberg, G. M., and Wang, S. S. (2006). Malleability of spiketiming-dependent plasticity at the
CA3-CA1 synapse. J. Neurosci. 26, 6610-6617.

Xu, T. X., and Yao, W. D. (2010). D1 and D2 dopamine receptors in separate circuits cooperate to drive associative long-term potentiation in the prefrontal cortex. Proc. Natl. Acad. Sci. U.S.A. 107, 16366-16371.

Yang, H. W., Lin, Y. W., Yen, C. D., and Min, M. Y. (2002). Change in bi-directional plasticity at CA1 synapses in hippocampal slices taken from 6-hydroxydopaminetreated rats: the role of endogenous norepinephrine. Eur. J. Neurosci. 16, 1117-1128.

Zhang, J. C., Lau, P. M., and Bi, G. Q. (2009). Gain in sensitivity and loss in temporal contrast of STDP by dopaminergic modulation at hippocampal synapses. Proc. Natl. Acad. Sci. U.S.A. 106, 13028-13033.

Conflict of Interest Statement: The authors declare that the research was conducted in the absence of any commercial or financial relationships that could be construed as a potential conflict of interest.

Received: 11 December 2012; paper pending published: 28 January 2013; accepted: 13 February 2013; published online: 06 March 2013

Citation: Edelmann $E$ and Lessmann $V$ (2013) Dopamine regulates intrinsic excitability thereby gating successful induction of spike timing-dependent plasticity in CA1 of the hippocampus. Front. Neurosci. 7:25. doi: 10.3389/fnins. 2013.00025

Copyright (C) 2013 Edelmann and Lessmann. This is an open-access article distributed under the terms of the Creative Commons Attribution License, which permits use, distribution and reproduction in other forums, provided the original authors and source are credited and subject to any copyright notices concerning any third-party graphics etc. 